\title{
Image Segmentation Based on Doubly Truncated Generalized Laplace Mixture Model and K Means Clustering
}

\author{
T. Jyothirmayi ${ }^{1}$, K. Srinivasa Rao ${ }^{2}$, P. Srinivasa Rao ${ }^{3}$, Ch. Satyanarayana ${ }^{4}$ \\ ${ }^{1}$ Department of Computer Science and Engineering, GITAM University, Visakhapatnam, Andhra Pradesh, India \\ ${ }^{2}$ Department of Statistics, Andhra University, Visakhapatnam, Andhra Pradesh, India \\ ${ }^{3}$ Department of Computer Science and Systems Engineering, Andhra University, Visakhapatnam, Andhra Pradesh, India. \\ ${ }^{4}$ Department of Computer Science and Engineering, JNTU-Kakinada, Andhra Pradesh, India
}

\begin{abstract}
Article Info
Article history:

Received Mar 30, 2016

Revised Jul 30, 2016

Accepted Aug 17, 2016

Keyword:

Double truncated generalized

Laplace mixture model

Generalized Laplace mixture model

Image segmentation algorithm

Performance measures

ABSTRACT

The present paper aims at performance evaluation of Doubly Truncated Generalized Laplace Mixture Model and K-Means clustering (DTGLMM-K) for image analysis concerned to various practical applications like security, surveillance, medical diagnostics and other areas. Among the many algorithms designed and developed for image segmentation the dominance of Gaussian Mixture Model (GMM) has been predominant which has the major drawback of suiting to a particular kind of data. Therefore the present work aims at development of DTGLMM-K algorithm which can be suitable for wide variety of applications and data. Performance evaluation of the developed algorithm has been done through various measures like Probabilistic Rand index (PRI), Global Consistency Error (GCE) and Variation of Information (VOI). During the current work case studies for various different images having pixel intensities has been carried out and the obtained results indicate the superiority of the developed algorithm for improved image segmentation.
\end{abstract}

Copyright (C) 2016 Institute of Advanced Engineering and Science. All rights reserved.

Corresponding Author:

T. Jyothirmayi,

Department of Computer Science and Engineering,

GITAM University,

Visakhapatnam, Andhra Pradesh, India.

Email: koka.jyothirmayi@gmail.com

\section{INTRODUCTION}

Image segmentation aims at identifying the regions of interest in an image or annotating the data of an image. It is the process to classify an image into several clusters according to the feature of image. Image segmentation techniques are based on some pixel or region similarity measures in relation to their local neighborhood. Segmentation techniques are broadly classified as region based, edge based, threshold based and model based [1]-[4]. Among these model based segmentation algorithms are found to be efficient compared to other [5]. In model based, entire image is viewed as a collection of image regions and each image region is characterized by a probability distribution function of pixels.

The pixel intensity is considered as a feature component of the image. The pixel intensities in image may be meso kurtic, platy kurtic, lepto kurtic, symmetric and asymmetric. The efficiency of segmentation algorithm depends on probability distribution followed by the pixels in an image. Much work has been reported considering the pixel intensities follow a Gaussian distribution and variates of finite GMM.

Zhaoxia Fu et al [6] proposed an image segmentation method which used Gaussian Mixture Models to model the original image and transforms the segmentation problem into maximum likelihood parameter estimation by expectation-maximization(EM algorithm) and classify the pixels in image. Thanh Minh Nguyen et al [7] proposed a mixture model for image segmentation which incorporated spatial information between neighboring pixels into the Gaussian mixture model based on Markov random field (MRF). EM 
algorithm was applied to optimize the parameters. Nagesh V et al [8] suggested an approach for image segmentation of medical images based on Finite Truncated Skew Gaussian distribution. Vamsi Krishna $M$ et al [9] developed a segmentation model for brain images based on bivariate Gaussian distribution model with k-means clustering.

However application of finite GMM is accurate and successful for all kinds of pixel intensities except lepto kurtic data. Therefore an alternative image segmentation algorithm which can be applicable to all kinds of data is needed and the present work concentrates mainly in this aspect by means of generalization of GMM with respect to kurtosis. Jyothirmayi et al [10] developed and analyzed an image segmentation algorithm based on Generalized Laplace Mixture Model (GLMM) where the pixel intensities lie in range of $(-\infty, \infty)$. The developed model was integrated with hierarchical clustering method and used for image segmentation of images which are having platykurtic and lepto kurtic nature. Hierarchical clustering and moment method of estimation was used for initialization of parameters.

As an extension to the previous works, within the current work an attempt is made to extend the GLMM as Doubly Truncated GLMM (DTGLMM) by truncating the range of pixel intensity values within a specified range. K-means algorithm is used for initialization of parameters since it is simple and works well for large data sets when compared with hierarchical clustering. Performance evaluation of the developed model has been carried out by means of analysis of various different categories of images as case studies.

\section{PROPOSED METHOD}

In this paper an algorithm DTGLMM-K has been proposed for image segmentation. It is assumed that the whole image is collection of image regions in which the pixel intensity of each region follows a generalized laplace distribution. The parameters mean, variance of DTGLMM are estimated through EM algorithm. Initialization of the parameters is obtained by K-Means clustering. Image analysis with the developed algorithm is performed on five images from Berkeley image data set and compared with existing algorithms available in the literature.

\subsection{Doubly Truncated Generalized Laplace Distribution}

Image segmentation algorithms consider image as a collection of image regions where each image region is represented by pixel intensities. For a given point pixel $(x, y)$, the pixel intensity $\mathrm{z}=\mathrm{f}(\mathrm{x}, \mathrm{y})$ is a random variable. It is generallyassumed that the pixel intensities within the region lie in an infinite range. Assuming that the pixel intensity lie between ' $a$ ' and ' $b$ ', the probability density function of the pixel intensity is given by

$$
\mathrm{f}(\mathrm{x}, \mu, \sigma 2)=\left(\frac{\left(\mathrm{r}^{2}+\frac{(\mathrm{x}-\mu)^{2}}{\sigma^{2}}\right)^{\mathrm{r}} \mathrm{e}^{-\left|\frac{\mathrm{x}-\mu}{\sigma}\right|}}{2 \sigma \sum_{\mathrm{k}=0}^{\mathrm{r}}\left(\begin{array}{l}
\mathrm{r} \\
\mathrm{k}
\end{array}\right) \mathrm{r}^{2(\mathrm{r}-\mathrm{k})}\left(\int_{\frac{\mathrm{a}-\mu}{\sigma}}^{\frac{\mathrm{b}-\mu}{\sigma}} \mathrm{x}^{2 \mathrm{k}} \mathrm{e}^{-|\mathrm{x}|} \mathrm{dx}\right)}\right) \text { where } \mathrm{a}<x<b, a<\mu<b, \sigma>0
$$

or

$$
f(x, \mu, \sigma 2)=\frac{\left(r^{2}+\frac{(x-\mu)^{2}}{\sigma^{2}}\right)^{r} e^{-\left|\frac{x-\mu}{\sigma}\right|}}{2 \sigma \sum_{k=0}^{r}\left(\begin{array}{l}
r \\
k
\end{array}\right) r^{2(r-k)}\left[\gamma\left[(2 k+1),-\left(\frac{a-\mu}{\sigma}\right)\right]+\gamma\left[(2 k+1),-\left(\frac{b-\mu}{\sigma}\right)\right]\right]}
$$

Here it is assumed that entire image is collection of several image regions. In each image region the pixel intensities are characterized by doubly truncated generalized Laplace probability model. The probability density function of pixel intensities in whole image is of the form

$$
p(x)=\sum_{i=1}^{k} \alpha_{i} f_{i}\left(x_{s}, \mu_{i}, \sigma_{i}^{2}\right)
$$

where $\mathrm{k}$ is the number of regions, $0 \leq \alpha \mathrm{i} \leq 1$ are weights such that $\Sigma \alpha_{\mathrm{i}}=1$ and $\alpha_{\mathrm{i}}$ is the weight associated with $\mathrm{i}^{\text {th }}$ region in the whole image and $f_{i}(x, \mu, \sigma 2)$ is the probability density function of Generalized Laplace distribution of $i^{\text {th }}$ image region and is as given in equation (1).

The mean of the distribution is 


$$
E(X)=\int_{a}^{b} x f(x) d x=\mu+\frac{\sum_{k=0}^{r}\left(\begin{array}{l}
r \\
k
\end{array}\right) r^{2(r-k)}\left[\gamma\left[(2 k+2),\left(\frac{b-\mu}{\sigma}\right)\right]-\gamma\left[(2 k+2),-\left(\frac{a-\mu}{\sigma}\right)\right]\right]}{\sum_{k=0}^{r}\left(\begin{array}{l}
r \\
k
\end{array}\right) r^{2(r-k)}\left[\gamma\left[(2 k+1),\left(\frac{b-\mu}{\sigma}\right)\right]-\gamma\left[(2 k+1),-\left(\frac{a-\mu}{\sigma}\right)\right]\right]}
$$

The variance of the distribution is $\operatorname{var}(\mathrm{x})=\operatorname{var}\left(\frac{1}{\mathrm{n}} \sum_{\mathrm{i}=1}^{\mathrm{n}} \mathrm{X}_{\mathrm{i}}\right)$

$$
=\frac{\frac{1}{n} \sum_{\mathbf{q}=0}^{2}\left(\begin{array}{l}
2 \\
\mathbf{q}
\end{array}\right)\left(\mu-\mu_{1}^{1}\right)^{2-\mathrm{q}} \sigma^{\mathrm{q}}\left[(-1)^{\mathrm{q}} \gamma\left[(\mathrm{q}+1),-\left(\frac{\mathrm{a}-\mu}{\sigma}\right)\right]+(-1)^{\mathrm{q}} \gamma\left[(\mathrm{q}+3),-\left(\frac{\mathrm{a}-\mu}{\sigma}\right)\right]+\gamma\left[(\mathrm{q}+1),-\left(\frac{\mathrm{b}-\mu}{\sigma}\right)\right]+\gamma\left[(\mathrm{q}+3),-\left(\frac{\mathrm{b}-\mu}{\sigma}\right)\right]\right]}{\sum_{\mathrm{k}=0}^{\mathrm{r}}\left(\begin{array}{l}
\mathrm{r} \\
\mathrm{k}
\end{array}\right) \mathrm{r}^{2(\mathrm{r}-\mathrm{k})}\left[\gamma\left[(2 \mathrm{k}+1),-\left(\frac{\mathrm{a}-\mu}{\sigma}\right)\right]-\gamma\left[(2 \mathrm{k}+1),\left(\frac{\mathrm{b}-\mu}{\sigma}\right)\right]\right]}
$$

\subsection{Estimation Of The Model Parameters By EM Algorithm}

The estimates of the model parameters are obtained through EM algorithm. To obtain accurate result it is considered that the range of pixel intensities is finite in nature and doubly truncated generalized Laplace distribution is well suited. Its probability distribution function is given in equation 2 .

The likelihood function of observations $\mathrm{x} 1, \mathrm{x} 2 \ldots \mathrm{xn}$ is $\mathrm{L}(\theta)=\prod_{s=1}^{\mathrm{N}} \mathrm{p}\left(\mathrm{x}_{\mathrm{s}}, \theta^{\mathrm{l}}\right)$

$$
\begin{aligned}
& \text { (i.e) } \mathrm{L}(\theta)=\prod_{\mathrm{s}=1}^{\mathrm{N}}\left(\sum_{\mathrm{i}=1}^{\mathrm{k}} \alpha_{\mathrm{i}} \mathrm{f}_{\mathrm{i}}\left(\mathrm{x}_{\mathrm{s}}, \mu_{\mathrm{i}}, \sigma_{\mathrm{i}}^{2}\right)\right) \\
& \log \mathrm{L}(\theta)=\sum_{\mathrm{s}=1}^{\mathrm{N}} \log \left[\left(\frac{\left(\mathrm{r}^{2}+\frac{(\mathrm{x}-\mu)^{2}}{\sigma^{2}}\right)^{\mathrm{r}} \mathrm{e}^{-\left|\frac{\mathrm{x}-\mu}{\sigma}\right|}}{2 \sigma \sum_{\mathrm{k}=0}^{\mathrm{r}}\left(\begin{array}{c}
\mathrm{r} \\
\mathrm{k}
\end{array}\right) \mathrm{r}^{2(\mathrm{r}-\mathrm{k})}\left(\frac{\int_{-}^{\frac{\mathrm{b}-\mu}{\sigma}}}{\sigma} \mathrm{x}^{2 \mathrm{k}} \mathrm{e}^{-|\mathrm{x}|} \mathrm{dx}\right)}\right)\right]
\end{aligned}
$$

The updated equations of EM algorithm for estimating the model parameters are

$$
\frac{\partial}{\partial \mu \mathrm{i}}\left[\sum_{\mathrm{i}=1}^{\mathrm{k}} \sum_{\mathrm{s}=1}^{\mathrm{N}} \mathrm{T}_{\mathrm{i}}\left(\mathrm{x}_{\mathrm{s}}, \theta^{\mathrm{l}}\right)\left[\log \left[\left(\frac{\left(\mathrm{r}^{2}+\frac{(\mathrm{x}-\mu)^{2}}{\sigma^{2}}\right)^{\mathrm{r}} \mathrm{e}^{-\left|\frac{\mathrm{x}-\mu}{\sigma}\right|}}{2 \sigma \sum_{\mathrm{k}=0}^{\mathrm{r}}\left(\begin{array}{l}
\mathrm{r} \\
\mathrm{k}
\end{array}\right) \mathrm{r}^{2(\mathrm{r}-\mathrm{k})}\left(\int_{\frac{\mathrm{a}-\mu}{\sigma}}^{\frac{\mathrm{b}-\mu}{\sigma}} \mathrm{x}^{2 \mathrm{k}} \mathrm{e}^{-|\mathrm{x}|} \mathrm{dx}\right)}\right)\right]\right]+\log \left(\alpha_{\mathrm{i}}^{\mathrm{l}}\right)\right]=0
$$

This implies

$$
\begin{aligned}
& \sum_{s=1}^{N} \mathrm{~T}_{\mathrm{i}}\left(\mathrm{x}_{\mathrm{s}}, \theta^{\mathrm{l}}\right)\left[\left(\frac{\left(\mathrm{x}_{\mathrm{s}}-\mu_{\mathrm{i}}\right)}{\sigma_{\mathrm{i}}{ }^{2}}\right)\left[\frac{\left[-2 \sigma_{\mathrm{i}}{ }^{2}\right]}{\mathrm{r}^{2} \sigma_{\mathrm{i}}{ }^{2}\left(\mathrm{x}_{\mathrm{s}}-\mu_{\mathrm{i}}\right)^{2}}+\frac{\sigma_{\mathrm{i}}}{\left|\mathrm{x}_{\mathrm{s}}-\mu_{\mathrm{i}}\right|}\right]\right]=0 \\
& \sum_{s=1}^{\mathrm{N}} \mathrm{T}_{\mathrm{i}}\left(\mathrm{x}_{\mathrm{s}}, \theta^{\mathrm{l}}\right) \frac{\mathrm{x}_{\mathrm{s}}-\mu_{\mathrm{i}}}{\sigma_{\mathrm{i}}\left|\mathrm{x}_{\mathrm{s}}-\mu_{\mathrm{i}}\right|}-\sum_{\mathrm{s}=1}^{\mathrm{N}} \mathrm{T}_{\mathrm{i}}\left(\mathrm{x}_{\mathrm{s}}, \theta^{\mathrm{l}}\right) \frac{\left[2\left(\mathrm{x}_{\mathrm{s}}-\mu_{\mathrm{i}}\right)\right]}{\mathrm{r}^{2} \sigma_{\mathrm{i}}{ }^{2}+\left(\mathrm{x}_{\mathrm{s}}-\mu_{\mathrm{i}}\right)^{2}}=0
\end{aligned}
$$

where $T_{i}\left(x_{S}, \theta^{l}\right)=\frac{\alpha_{i}^{l+1} f_{i}\left(x_{s}, \theta^{l}\right)}{\sum_{i=1}^{k} \alpha_{i}^{1+1} f_{i}\left(x_{s}, \theta^{l}\right)}$

For updating $\sigma_{\mathrm{i}}^{2}$ differentiate $\mathrm{Q}\left(\theta ; \theta^{\mathrm{l}}\right)$ w.r.t ${\sigma_{\mathrm{i}}}^{2}$ and equate to 0

$$
\begin{aligned}
& \frac{\partial}{\partial \sigma_{\mathrm{i}}{ }^{2}} \mathrm{Q}\left(\theta ; \theta^{\mathrm{l}}\right)=0 \\
& \frac{\partial}{\partial \sigma_{\mathrm{i}}{ }^{2}}\left[\sum _ { \mathrm { i } = 1 } ^ { \mathrm { k } } \sum _ { \mathrm { s } = 1 } ^ { \mathrm { N } } \mathrm { T } _ { \mathrm { i } } ( \mathrm { x } _ { \mathrm { s } } , \theta ^ { \mathrm { l } } ) \left[\log \left[\left(\frac{\left(\mathrm{r}^{2}+\frac{(\mathrm{x}-\mu)^{2}}{\sigma^{2}}\right)^{\mathrm{r}} \mathrm{e}^{-\left|\frac{\mathrm{x}-\mu}{\sigma}\right|}}{\left.2 \sigma \sum_{\mathrm{k}=0}^{\mathrm{r}}\left(\begin{array}{l}
\mathrm{r} \\
\mathrm{k}
\end{array}\right) \mathrm{r}^{2(\mathrm{r}-\mathrm{k})}\left(\frac{\left.\int^{\frac{\mathrm{a}-\mu}{\sigma}} \mathrm{x}^{2 \mathrm{k}} \mathrm{e}^{-|\mathrm{x}|} \mathrm{dx}\right)}{\sigma}\right)\right]}\right]+\log \left(\alpha_{\mathrm{i}}^{\mathrm{l}}\right)\right]=0\right.\right.
\end{aligned}
$$




$$
\begin{aligned}
& \sum_{\mathrm{s}=1}^{\mathrm{N}}\left[\frac{\left(\mathrm{x}_{\mathrm{s}}-\mu_{\mathrm{i}}\right)^{2}}{\left(\mathrm{r}^{2} \sigma_{\mathrm{i}}^{2}+\left(\mathrm{x}_{\mathrm{s}}-\mu_{\mathrm{i}}\right)^{2}\right) \sigma_{\mathrm{i}}{ }^{2}}-\left|\frac{\mathrm{x}_{\mathrm{s}}-\mu_{\mathrm{i}}}{2 \sigma_{\mathrm{i}}{ }^{3}}\right|-\frac{1}{2 \sigma_{\mathrm{i}}{ }^{2}}\right. \\
& \left.-\frac{\sum_{\mathrm{k}=0}^{\mathrm{r}}\left(\begin{array}{l}
\mathrm{r} \\
\mathrm{k}
\end{array}\right) \mathrm{r}^{2(\mathrm{r}-\mathrm{k})}\left[\left(\frac{\mathrm{b}-\mu_{\mathrm{i}}}{\sigma_{\mathrm{i}}}\right)^{2 \mathrm{k}} \cdot \frac{\mathrm{b}-\mu_{\mathrm{i}}}{\sigma_{\mathrm{i}}{ }^{3}} \cdot \mathrm{e}^{-\left|\frac{\mathrm{b}-\mu}{\sigma}\right|}-\left(\frac{\mathrm{a}-\mu_{\mathrm{i}}}{\sigma_{\mathrm{i}}}\right)^{2 \mathrm{k}} \cdot \frac{\mathrm{a}-\mu_{\mathrm{i}}}{\sigma_{\mathrm{i}}{ }^{3}} \cdot \mathrm{e}^{-\left|\frac{\mathrm{a}-\mu}{\sigma}\right|}\right]}{\sum_{\mathrm{k}=0}^{\mathrm{r}}\left(\begin{array}{l}
\mathrm{r} \\
\mathrm{k}
\end{array}\right) \mathrm{r}^{2(\mathrm{r}-\mathrm{k})}\left(\frac{\frac{\mathrm{b}-\mu}{\sigma}}{\sigma} \mathrm{x}^{2 \mathrm{k}} \mathrm{e}^{-|\mathrm{x}|} \mathrm{dx}\right.}\right] \mathrm{T}_{\mathrm{i}}\left(\mathrm{x}_{\mathrm{s}}, \theta^{\mathrm{l}}\right)=0 \\
& \text { where } \mathrm{T}_{\mathrm{i}}\left(\mathrm{x}_{\mathrm{s}}, \theta^{\mathrm{l}}\right)=\frac{\alpha_{\mathrm{i}}{ }^{\mathrm{l}+1} \mathrm{f}_{\mathrm{i}}\left(\mathrm{x}_{\mathrm{s}}, \theta^{\mathrm{l}}\right)}{\sum_{\mathrm{i}=1}^{\mathrm{k}} \alpha_{\mathrm{i}}^{\mathrm{l}+1} \mathrm{f}_{\mathrm{i}}\left(\mathrm{x}_{\mathrm{s}}, \theta^{\mathrm{l}}\right)}
\end{aligned}
$$

solving the equations 7 and 8 we can get the final estimates of the parameters $\mu_{\mathrm{i}}$ and $\sigma_{\mathrm{i}}^{2}$.

\subsection{Initialization Of The Parameters By K Means Algorithm}

For EM algorithm the parameter $\alpha_{i}$ and the model parameters $\mu_{\mathrm{i}}$ and $\sigma_{\mathrm{i}}{ }^{2}$ have to be initialized which are usually considered as known. The initial value for $\alpha_{\mathrm{i}}=1 / \mathrm{k}$ where $\mathrm{k}$ is the number of image regions. The $\mathrm{k}$ value is initialized with number of peaks in histogram of image. After obtaining " $k$ " value in doubly truncated generalized Laplace model the estimation of model parameters is done by EM algorithm. The initial estimates of model parameters are obtained through K-Means algorithm and moment method of estimators for doubly truncated generalized Laplace mixture model. The shape parameter $\mathrm{r}$ can be estimated by sample kurtosis using the following equation

$$
\begin{aligned}
& \beta_{2}=\frac{\sum_{\mathrm{k}=0}^{\mathrm{r}}\left(\begin{array}{l}
\mathrm{r} \\
\mathrm{k}
\end{array}\right) \mathrm{r}^{2(\mathrm{r}-\mathrm{k})} \sum_{\mathrm{q}=0}^{4}\left(\begin{array}{l}
4 \\
\mathrm{q}
\end{array}\right)\left(\mu-\mu_{1}^{1}\right)^{4-\mathrm{q}_{\sigma} \mathrm{q}}\left[(-1)^{2 \mathrm{k}+\mathrm{q}} \gamma\left[(2 \mathrm{k}+\mathrm{q}+1),-\left(\frac{\mathrm{a}-\mu}{\sigma}\right)\right]+\gamma\left[(2 \mathrm{k}+\mathrm{q}+1),\left(\frac{\mathrm{b}-\mu}{\sigma}\right)\right]\right]}{\left[\sum_{\mathrm{k}=0}^{\mathrm{r}}\left(\begin{array}{l}
\mathrm{r} \\
\mathrm{k}
\end{array}\right) \mathrm{r}^{2(\mathrm{r}-\mathrm{k})} \sum_{\mathrm{q}=0}^{4}\left(\begin{array}{l}
2 \\
\mathrm{q}
\end{array}\right)\left(\mu-\mu_{1}^{1}\right)^{2-\mathrm{q}_{\sigma} \mathrm{q}}\left[(-1)^{2 \mathrm{k}+\mathrm{q}} \gamma\left[(2 \mathrm{k}+\mathrm{q}+1),-\left(\frac{\mathrm{a}-\mu}{\sigma}\right)\right]+\gamma\left[(2 \mathrm{k}+\mathrm{q}+1),\left(\frac{\mathrm{b}-\mu}{\sigma}\right)\right]\right]\right]^{2}} \sum_{\mathrm{k}=0}^{\mathrm{r}}\left(\begin{array}{l}
\mathrm{r} \\
\mathrm{k}
\end{array}\right) \mathrm{r}^{2(\mathrm{r}-\mathrm{k})} \gamma[(2 \mathrm{k}+ \\
& \left.1),-\left(\frac{\mathrm{a}-\mu}{\sigma}\right)\right]+\gamma\left[(2 \mathrm{k}+1),\left(\frac{\mathrm{b}-\mu}{\sigma}\right)\right]
\end{aligned}
$$

The mean of the distribution is $\overline{\mathrm{x}}=\mu+\frac{\sum_{\mathrm{k}=0}^{\mathrm{r}}\left(\begin{array}{l}\mathrm{r} \\ \mathrm{k}\end{array}\right) \mathrm{r}^{2(\mathrm{r}-\mathrm{k})}\left[\gamma\left[(2 \mathrm{k}+2),\left(\frac{\mathrm{b}-\mu}{\sigma}\right)\right]-\gamma\left[(2 \mathrm{k}+2),-\left(\frac{\mathrm{a}-\mu}{\sigma}\right)\right]\right]}{\sum_{\mathrm{k}=0}^{\mathrm{r}}\left(\begin{array}{l}\mathrm{r} \\ \mathrm{k}\end{array}\right) \mathrm{r}^{2(\mathrm{r}-\mathrm{k})}\left[\gamma\left[(2 \mathrm{k}+1),\left(\frac{\mathrm{b}-\mu}{\sigma}\right)\right]-\gamma\left[(2 \mathrm{k}+1),-\left(\frac{\mathrm{a}-\mu}{\sigma}\right)\right]\right]}$

and $\sigma 2=\frac{\frac{1}{\mathrm{n}} \sum_{\mathrm{q}=0}^{2}\left(\begin{array}{l}2 \\ \mathrm{q}\end{array}\right)\left(\mu-\mu_{1}^{1}\right)^{2-\mathrm{q}} \sigma^{\mathrm{q}}\left[(-1)^{\mathrm{q}} \gamma\left[(\mathrm{q}+1),-\left(\frac{\mathrm{a}-\mu}{\sigma}\right)\right]+(-1)^{\mathrm{q}} \gamma\left[(\mathrm{q}+3),-\left(\frac{\mathrm{a}-\mu}{\sigma}\right)\right]+\gamma\left[(\mathrm{q}+1),-\left(\frac{\mathrm{b}-\mu}{\sigma}\right)\right]+\gamma\left[(\mathrm{q}+3),-\left(\frac{\mathrm{b}-\mu}{\sigma}\right)\right]\right]}{\sum_{\mathrm{k}=0}^{\mathrm{r}}\left(\begin{array}{l}\mathrm{r} \\ \mathrm{k}\end{array}\right) \mathrm{r}^{2}(\mathrm{r}-\mathrm{k})\left[\gamma\left[(2 \mathrm{k}+1),-\left(\frac{\mathrm{a}-\mu}{\sigma}\right)\right]-\gamma\left[(2 \mathrm{k}+1),\left(\frac{\mathrm{b}-\mu}{\sigma}\right)\right]\right]}$

with known values of $\mathrm{a}$ and $\mathrm{b}$ solving equations 9,10 and 11 simultaneously by Newton Raphson Method the parameters $\mu_{\mathrm{i}}$ and $\sigma_{\mathrm{i}}^{2}$ are obtained. With these initial estimates final estimates are obtained through EM algorithm as given in section 3 .

\subsection{Segmentation Algorithm}

After refining the parameters the next step is to segment the image by allocating the pixels to the segments. This operation is performed by segmentation algorithm. The image segmentation algorithm consists of four steps.

Step 1: Using K-Means algorithm attain the number of image regions.

Step 2: Acquire the initial estimates of the model parameters using K-Means algorithm.

Step 3: Using the EM algorithm with updated equations, find the refined estimates of the model parameters $\alpha \mathrm{i}, \mu \mathrm{i}, \sigma \mathrm{i} 2$ for $\mathrm{i}=1,2 \ldots . \mathrm{k}$.

Step 4: Allocate each pixel into the corresponding $\mathrm{j}^{\text {th }}$ region according to the maximum likelihood of the $\mathrm{jth}$ component $\mathrm{Lj}$.

That is $z_{s}$ is assigned to the $j^{\text {th }}$ region for which $L$ is maximum.

$$
\mathrm{L}=\max \left(\frac{\left(\mathrm{r}^{2}+\frac{(\mathrm{x}-\mu)^{2}}{\sigma^{2}}\right)^{\mathrm{r}} \mathrm{e}^{-\left|\frac{\mathrm{x}-\mu}{\sigma}\right|}}{2 \sigma \sum_{\mathrm{k}=0}^{\mathrm{r}}\left(\begin{array}{l}
\mathrm{r} \\
\mathrm{k}
\end{array}\right) \mathrm{r}^{2(\mathrm{r}-\mathrm{k})}\left(\int_{\frac{\mathrm{a}-\mu}{\sigma}}^{\frac{\mathrm{b}-\mu}{\sigma}} \mathrm{x}^{2 \mathrm{k}} \mathrm{e}^{-|\mathrm{x}|} \mathrm{dx}\right)}\right)
$$




\section{RESULTS AND DISCUSSION}

For conducting the experimentation randomly five images were taken from Berkeley image dataset (www.eecs.berkeley.edu/Research/Projects/CS/vision/bsds/BSDS300/html/dataset/images.html). Image consists of $\mathrm{K}$ image regions and initial value of $\mathrm{K}$ is obtained by histogram of pixel intensities. The five images and their respective histograms are shown in Figure 1.

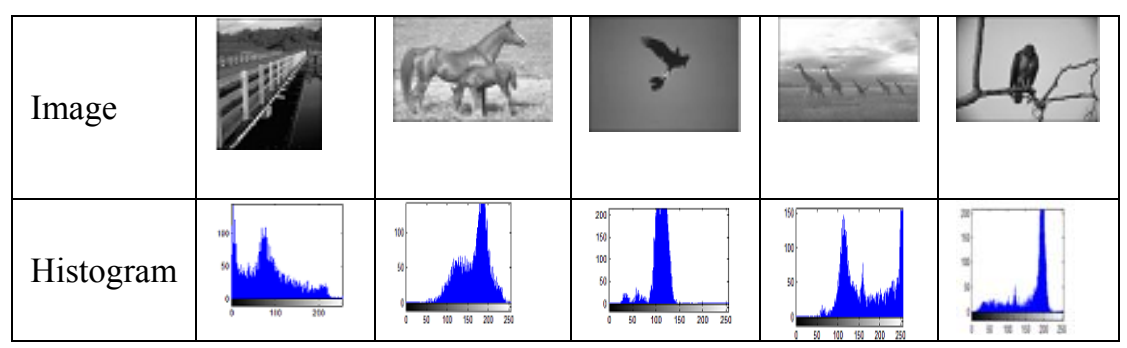

Figure 1. Images and corresponding Histograms

Based on histograms the initial estimates for five images have been obtained. The model parameters considered are $\alpha_{i}, \mu_{i}$ and $\sigma_{i}$ for $i=1,2 \ldots K$ where $\mathrm{K}$ is the number of regions and they are obtained by the method given in section 2.3. Final parameters for the five images have been derived using these initial parameters and updated equations in section 2.2 and the final parameters and presented in Tables 1,2,3,4 and 5.

Table 1. Estimation of parameters for Image 1

\begin{tabular}{|c|c|c|c|c|c|c|}
\hline \multicolumn{7}{|c|}{ Estimated Values of the Parameters for Image1 Number of regions $(\mathrm{K}=3)$} \\
\hline \multirow{2}{*}{ Parameters } & \multicolumn{3}{|c|}{ Estimation of Initial Parameters By K Means } & \multicolumn{3}{|c|}{ Estimation of Final Parameters by EM Algorithm } \\
\hline & Region1 & Region2 & Region3 & Region1 & Region2 & Region3 \\
\hline$\alpha_{i}$ & 0.33 & 0.33 & 0.33 & 0.0 & 0.0499 & 0.9501 \\
\hline$\mu_{\mathrm{i}}$ & 19.34 & 85.56 & 173.93 & 15.5 & 63.23 & 107.89 \\
\hline$\sigma_{i}$ & 15.86 & 19.45 & 30.37 & 8.53 & 17.30 & 27.26 \\
\hline \multicolumn{4}{|c|}{$\mathrm{a}=0$} & & $b=255$ & \\
\hline
\end{tabular}

Table 2. Estimation of parameters for Image 2

\begin{tabular}{|c|c|c|c|c|c|c|}
\hline \multicolumn{7}{|c|}{ Estimated Values of the Parameters for Image 2 Number of regions $(K=3)$} \\
\hline \multirow{2}{*}{ Parameters } & \multicolumn{3}{|c|}{ Estimation of Initial Parameters By K Means } & \multicolumn{3}{|c|}{ Estimation of Final Parameters by EM Algorithm } \\
\hline & Region1 & Region2 & Region3 & Region1 & Region2 & Region3 \\
\hline$\alpha_{i}$ & 0.33 & 0.33 & 0.33 & 0.19 & 0.35 & 0.45 \\
\hline$\mu_{\mathrm{i}}$ & 198.65 & 165.48 & 113.61 & 173.61 & 142.16 & 93.80 \\
\hline \multirow[t]{2}{*}{$\sigma_{\mathrm{i}}$} & 13.96 & 12.77 & 18.88 & 13.53 & 18.3 & 18.2 \\
\hline & $\mathrm{a}=34$ & & \multicolumn{4}{|c|}{$b=254$} \\
\hline
\end{tabular}

Table 3. Estimation of parameters for Image 3

\begin{tabular}{|c|c|c|c|c|c|c|}
\hline \multicolumn{7}{|c|}{ Estimated Values of the Parameters for Image 3 Number of regions $(\mathrm{K}=3)$} \\
\hline \multirow{2}{*}{ Parameters } & \multicolumn{3}{|c|}{ Estimation of Initial Parameters By K Means } & \multicolumn{3}{|c|}{ Estimation of Final Parameters by EM Algorithm } \\
\hline & Region1 & Region2 & Region3 & Region1 & Region2 & Region3 \\
\hline$\alpha_{\mathrm{i}}$ & 0.33 & 0.33 & 0.33 & -0.08 & -0.12 & 1.2 \\
\hline$\mu_{\mathrm{i}}$ & 103.69 & 52.32 & 122.42 & 110.55 & 49.37 & 134.80 \\
\hline$\sigma_{\mathrm{i}}$ & 6.66 & 15.36 & 6.88 & 8.53 & $\begin{array}{c}8.3 \\
b=187\end{array}$ & 8.26 \\
\hline
\end{tabular}

Table 4. Estimation of parameters for Image4

\begin{tabular}{|c|c|c|c|c|c|c|}
\hline \multicolumn{7}{|c|}{ Estimated Values of the Parameters for Image 4 Number of regions $(\mathrm{K}=3)$} \\
\hline \multirow{2}{*}{ Parameters } & \multicolumn{3}{|c|}{ Estimation of Initial Parameters By K Means } & \multicolumn{3}{|c|}{ Estimation of Final Parameters by EM Algorithm } \\
\hline & Region1 & Region2 & Region3 & Region1 & Region2 & Region3 \\
\hline$\alpha_{\mathrm{i}}$ & 0.33 & 0.33 & 0.33 & 0.05 & 0.32 & 0.62 \\
\hline$\mu_{\mathrm{i}}$ & 113.39 & 172.35 & 241.02 & 193.86 & 180.16 & 129.80 \\
\hline$\sigma_{\mathrm{i}}$ & 16.42 & 18.72 & 14.30 & 10.09 & 13.7 & 10.27 \\
\hline \multicolumn{4}{|c|}{$a=30$} & & $b=255$ & \\
\hline
\end{tabular}


Table 5. Estimation of parameters for Image 5

\begin{tabular}{ccccccc}
\hline \multirow{2}{*}{ Parameters } & \multicolumn{3}{c}{ Estimated Values of the Parameters for Image5 Number of regions $(\mathrm{K}=3)$} \\
& Estimation of Initial Parameters By K Means & \multicolumn{2}{c}{ Estimation of Final Parameters by EM Algorithm } \\
& Region1 & Region2 & Region3 & Region1 & Region2 & Region3 \\
\hline$\alpha_{\mathrm{i}}$ & 0.33 & 0.33 & 0.33 & 0.3570 & 0.6037 & 0.0394 \\
$\mu_{\mathrm{i}}$ & 193.36 & 125.28 & 53.88 & 142.57 & 62.28 & 34.80 \\
$\sigma_{\mathrm{i}}$ & 10.28 & 19.88 & 20.26 & 10.53 & 18.30 & 18.22 \\
& & $\mathrm{a}=5$ & & & $\mathrm{~b}=231$ & \\
\hline
\end{tabular}

Substituting the final estimates of the model parameters the probability density function of the pixel intensities in each image are estimated.

The estimated probability density function of the pixel intensities of the image 1 is $f\left(x, \theta^{l}\right)=$

$$
\begin{gathered}
\frac{1}{34.12}\left(1+\frac{(x-15.5)^{2}}{8.53^{2}}\right)^{r} e^{-\left|\frac{x-15.5}{8.53}\right|}+\frac{1}{69.2}\left(1+\frac{(x-63.23)^{2}}{17.30^{2}}\right)^{r} e^{-\left|\frac{x-63.23}{17.30}\right|} \\
+\frac{1}{109.04}\left(1+\frac{(x-107.89)^{2}}{27.26^{2}}\right)^{r} e^{-\left|\frac{x-107.89}{27.26}\right|}
\end{gathered}
$$

The estimated probability density function of the pixel intensities of the image 2 is $f\left(x, \theta^{l}\right)=$

$$
\begin{gathered}
\frac{1}{27.06}\left(1+\frac{(x-173.61)^{2}}{13.53^{2}}\right)^{r} e^{-\left|\frac{x-173.61}{13.53}\right|}+\frac{1}{36.6}\left(1+\frac{(x-142.16)^{2}}{18.30^{2}}\right)^{r} e^{-\left|\frac{\mid x-142.16}{18.30}\right|} \\
+\frac{1}{36.4}\left(1+\frac{(x-93.80)^{2}}{18.2^{2}}\right)^{r} e^{-\left|\frac{x-93.80}{18.2}\right|}
\end{gathered}
$$

The estimated probability density function of the pixel intensities of the image 3 is $f\left(x, \theta^{l}\right)=$

$$
\begin{gathered}
\frac{1}{17.06}\left(1+\frac{(\mathrm{x}-110.55)^{2}}{8.53^{2}}\right)^{\mathrm{r}} \mathrm{e}^{-\left|\frac{\mathrm{x}-110.55}{8.53}\right|}+\frac{1}{16.6}\left(1+\frac{(\mathrm{x}-49.37)^{2}}{8.3^{2}}\right)^{\mathrm{r}} \mathrm{e}^{-\left|\frac{\mathrm{x}-49.37}{8.30}\right|} \\
+\frac{1}{16.52}\left(1+\frac{(\mathrm{x}-134.80)^{2}}{8.26^{2}}\right)^{\mathrm{r}} \mathrm{e}^{-\left|\frac{\mathrm{x}-134.8}{8.26}\right|}
\end{gathered}
$$

The estimated probability density function of the pixel intensities of the image 4 is $f\left(x, \theta^{l}\right)=$

$$
\begin{gathered}
\frac{1}{20.18}\left(1+\frac{(x-193.86)^{2}}{10.09^{2}}\right)^{r} e^{-\left|\frac{x-193.86}{10.09}\right|}+\frac{1}{27.4}\left(1+\frac{(x-180.16)^{2}}{13.7}\right)^{r} e^{-\left|\frac{x-180.16}{13.7}\right|} \\
+\frac{1}{20.54}\left(1+\frac{(x-129.80)^{2}}{10.27^{2}}\right)^{r} e^{-\left|\frac{x-129.80}{10.27}\right|}
\end{gathered}
$$

The estimated probability density function of the pixel intensities of the image 5 is $f\left(x, \theta^{l}\right)=$

$$
\begin{gathered}
\frac{1}{21.06}\left(1+\frac{(x-142.57)^{2}}{10.53^{2}}\right)^{r} e^{-\left|\frac{x-142.57}{10.53}\right|}+\frac{1}{36.60}\left(1+\frac{(x-62.28)^{2}}{18.30^{2}}\right)^{r} e^{-\left|\frac{x-62.28}{18.30}\right|} \\
+\frac{1}{36.44}\left(1+\frac{(x-34.80)^{2}}{18.22^{2}}\right)^{r} e^{-\left|\frac{x-34.80}{18.22}\right|}
\end{gathered}
$$

Using the probability density function and segmentation algorithm, segmentation is performed on following images. The original images and segmented images are shown in Figure 2. 


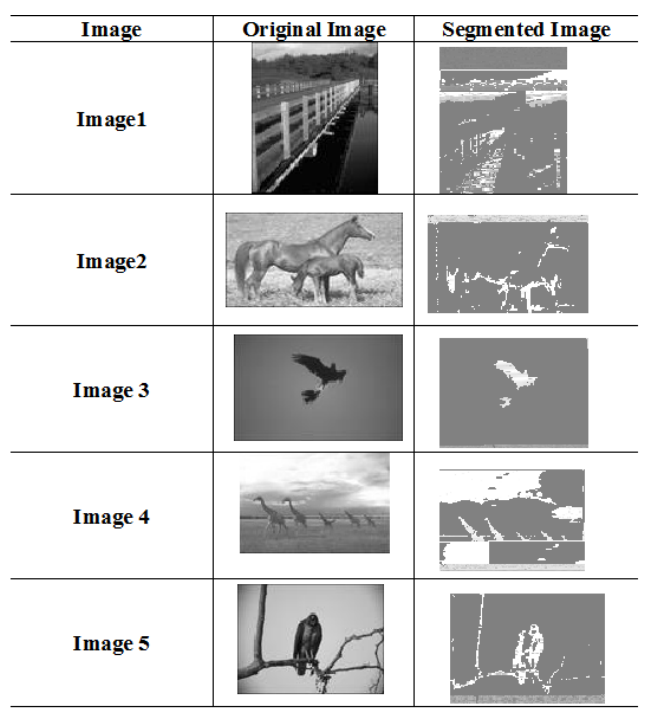

Figure 2. Original Image and Segmented Image

Once the image segmentation has been performed, its performance has been measured by calculating the performance metrics like Probabilistic Rand Index (PRI) given by Unnikrishnan R et al (2007), Global Consistency Error (GCE) given by Martin D. and et al and Variation of Information (VOI) given by Meila M (2005). The standard criteria for metrics is that PRI and GCE values must lie in range 0 to 1. The performance metrics for image segmentation method based on doubly truncated generalized mixture model using k-means is compared with GMM, GLMM using Hierarchical clustering (GLMM-H), and GLMM using K-Means clustering (GLMM-K) and shown in Figure 3. It is observed that the proposed method satisfies the standard criterion as the PRI and GCE values for five images are in range 0 to 1 and the proposed method outperforms the above methods.

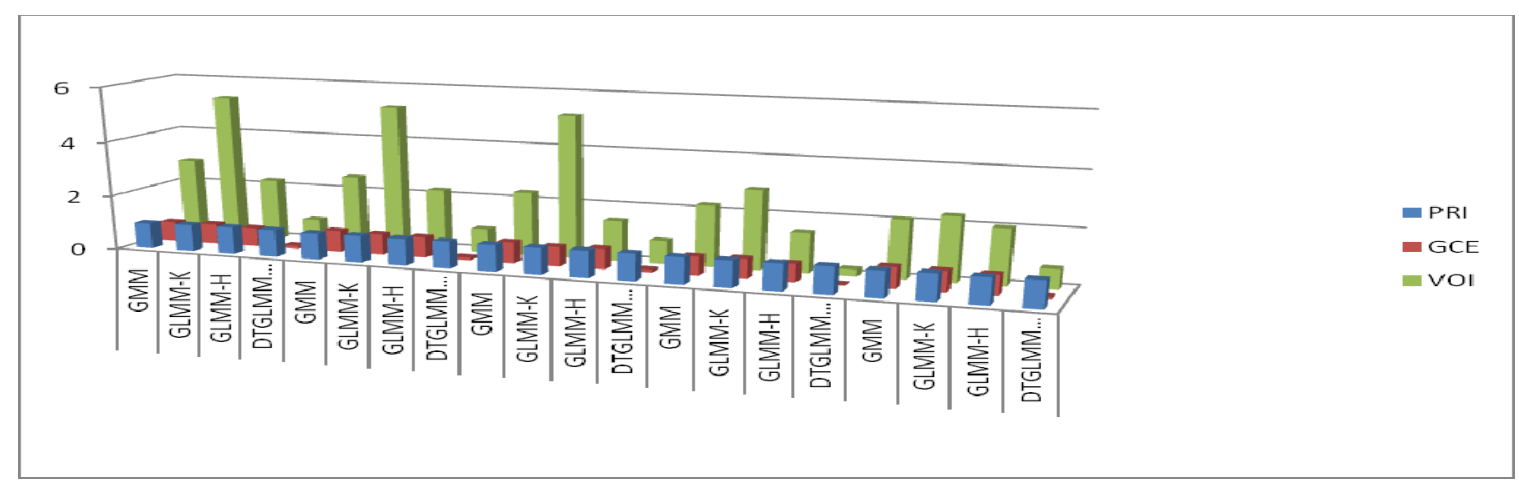

Figure 3. Comparison of Performance Measures

Using the developed algorithm the image can also be reconstructed. Once the image is reconstructed different quality metrics can be computed to study the performance of image quality. The quality metrics like image fidelity, mean square error and image quality have been calculated and shown in Table 6 as follows. 
Table 6. Segmentation Quality Metrics

\begin{tabular}{ccccc}
\hline \multirow{2}{*}{ Image } & \multirow{2}{*}{ Method } & Image Fidelity & Image Quality Metrics & \\
& & Signal to Noise Ratio & Image Quality Index \\
\hline Image1 & GMM & 0.99 & 0.24 & 1.0 \\
& GLMM-H & 0.99 & 0.24 & 0.988 \\
& GLMM-K & 0.99 & 0.419 & 0.988 \\
& DTGLMM-K & .99 & 0.34 & .99 \\
Image2 & GMM & 0.99 & 2.01 & 0.99 \\
& GLMM-K & 0.99 & 0.41 & 0.99 \\
& GLMM-H & 0.99 & 2.03 & 0.99 \\
& DTGLMM-K & .99 & 3.34 & .99 \\
Image3 & GMM & 0.98 & 1.45 & 0.98 \\
& GLMM-K & 0.99 & 0.03 & 0.99 \\
& GLMM-H & 0.99 & 1.45 & 0.99 \\
& DTGLMM-K & 0.99 & 2.25 & .98 \\
& GMM & .99 & 2.23 & .99 \\
& GLMM-K & .98 & 4.4 & 1.0 \\
& GLMM-H & .99 & 2.69 & .99 \\
& DTGLMM-K & 1.0 & 4.5 & 0.99 \\
& GMMM & .99 & 1.09 & .99 \\
& GLMM-K & .99 & 2.67 & .997 \\
& GLMM-H & .99 & 1.13 & .99 \\
\hline
\end{tabular}

From Table 6, it is observed that the images reconstructed using current method are close to reality. The image fidelity is almost same for all methods. The signal to noise ratio and image quality index is larger than other methods. The original images and reconstructed images using developed segmentation algorithm are presented in Figure 4.

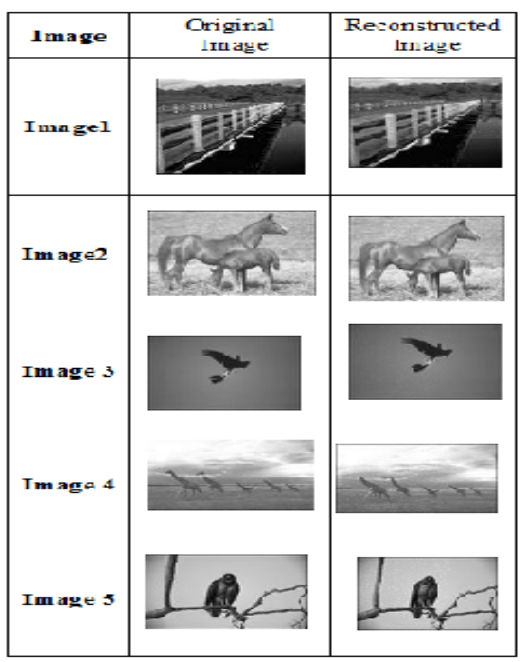

Figure 4. Original and Reconstructed Images

\section{CONCLUSION}

This paper discusses the development and analysis of image segmentation algorithm based on Doubly Truncated Generalized Laplace Mixture distribution. In many image segmentation algorithm it is customary to assume that pixel intensities associated with image regions are meso kurtic and having infinite range. But in reality in many images pixel intensities associated with image regions may not be meso kurtic and have infinite range. These two drawbacks of existing model based image segmentation algorithms are avoided in this paper by characterizing pixel intensity associated with image region follows Doubly Truncated Generalized Laplace distribution. The Doubly Truncated Generalized Laplace distribution is capable of portraying the image regions which are having symmetric or skewed and platy or meso kurtic pixel intensity distributions. The model parameters are obtained deriving updated equations for the doubly truncated generalized Laplace mixture model of EM algorithm. The initialization of model parameter is carried using K Means and moment method of estimation. The image segmentation algorithm is developed 
using maximum likelihood under Bayesian frame. The performance of the algorithm is evaluated through experimentation with five randomly images from Berkely data set and found that this method outperforms existing image segmentation method with image metrics PRI, GCE and VOI. This segmentation is much useful for image analysis of several images. The image segmentation methods can be further extended by considering doubly truncated multivariate generalized laplace mixture model which are useful for color images. This will be taken up in our further investigations. It is also observed the truncation of probability model significantly influence the segmentation performance.

\section{REFERENCES}

[1] W. X. Guang, et al., "An Improved Image Segmentation Algorithm Based on Two-Dimensional Otsu Method," Information Science Letters An International Journal, vol. 2, pp. 77-83, 2012.

[2] L. Zhengzhou, et al., "Gray-scale Edge Detection and Image Segmentation Algorithm Based on Mean Shift," Telkomnika Indonesian Journal of Electrical Engineering, vol/issue: 11(3), pp. 1414 -1421, 2013.

[3] M. Nahar, et al., "An Improved Approach for Digital Image Edge Detection," International Journal of Recent Development in Engineering and Technology, vol/issue: 2(3), 2014.

[4] R. Akhavan, et al., "A Novel Retinal Blood Vessel Segmentation Algorithm using Fuzzy segmentation," International Journal of Electrical and computer Engineering (IJECE), vol/issue: 4(4), pp. 561-572, 2014

[5] Suetens, et al., "Model-Based Image Segmentation: Methods and Applications," Lecture Notes in Medical Informatics, vol. 44, pp. 3-24.

[6] Z. Fu, et al., "Color Image Segmnetation using Gaussian Mixture Model and EM Algorithm," Multimedia and Signal Processing CCIS 346 Springer, pp. 61-66, 2012.

[7] T. M. Nguyen, et al., "An Extension of the standard Mixture Model for image segmentation," IEEE Transactions on Neural Networks, vol/issue: 21(8), 2010.

[8] N. Vadaparthi, et al., "An efficient approach for Medical Image segmentation Based on Truncated Skew Gaussian Mixture Model Using K-Means Algorithm," International Journal of Computer Science and Telecommunications, vol/issue: 2(6), pp. 81-88, 2011.

[9] M. V. Krishna, et al., "Bivariate Gaussian Mixture Model Based Segmentation for Effective Identification of Sclerosis in Brain MRI Images," International Journal of Engineering and Technical Research, vol/issue: 3(1), pp. 151-154, 2015.

[10] T. Jyothirmayi, et al., "Studied on Image Segmentation Integrating Generalized Laplace Mixture Model and Hierarchical Clustering," International Journal of Computer Applications, vol/issue: 128(12), 2015. 